\title{
The Clinical Meaning of Histamine Skin Reactivity
}

Roberto Ronchetti ${ }^{1}$ and Mario Barreto ${ }^{2 *}$

${ }^{1}$ Professor Emeritus of Pediatrics, "Sapienza" University, Rome, Italy

${ }^{2}$ Pediatric Unit of Sant'Andrea Hospital, NESMOS Department, Faculty of Medicine and Psychology, "Sapienza" University, Rome, Italy

"Corresponding author: Mario Barreto, Pediatric Unit of Sant'Andrea Hospital, NESMOS Department, "Sapienza" University, Rome, Italy, Tel: +39/0633775279; Fax: +39/0633775941; E-mail: mario.barreto@uniroma1.it

Received date: December 10, 2015; Accepted date: December 23, 2015; Published date: December 30, 2015

Copyright: ( 2015 Barreto M, et al. This is an open-access article distributed under the terms of the Creative Commons Attribution License, which permits unrestricted use, distribution, and reproduction in any medium, provided the original author and source are credited.

\begin{abstract}
The definition of the "atopic state", i.e. subjects presenting at least one skin wheal with a minimum diameter of 3 $\mathrm{mm}$ induced by an allergen skin-prick test (ASPT), is based on the assumption that wheal size depends entirely on the amount of histamine produced in the antigen-antibody reaction. Several epidemiological studies have, however, demonstrated that an ASPT-elicited wheal is heavily modulated by "histamine skin reactivity" (HSR), i.e. the size of the wheal induced by a prick test performed with a given solution of histamine. HSR not only varies widely depending on the individual characteristics and geographical setting, but also changes over time; these differences in HSR markedly influence the amount of specific IgE required to produce a wheal of at least $3 \mathrm{~mm}$ in an ASPT. We should therefore ideally conceive the existence of two types of" atopic patients": one type in whom "atopy" is mainly the result of an increased level of specific lgE antibodies, and another type in whom positive ASPTs are mainly the result of marked skin reactivity to even small amounts of histamine. If hyper-reactivity to histamine occurs not only in the skin but in parallel also in other parts of the organism, especially at the mucosal level, "normal" histamine production may cause chronic or recurrent clinical symptoms.
\end{abstract}

Keywords:

Histamine skin reactivity; Allergen skin-prick test; Atopy; Children; Antigen; Immunoglobulin E; Wheal size

\section{Background}

An atopic state in a subject is defined as a skin wheal of at least 3 $\mathrm{mm}$ in diameter elicited by an allergen skin prick test (ASPT) performed with common environmental allergens (Dermatophagoides, pollens, cat, etc.) $[1,2]$.

The $3 \mathrm{~mm}$ threshold of significance of the wheal is a mere convention adopted for practical purposes: a $4-10 \mathrm{~mm}$ wheal characterizes highly atopic subjects, while a 1-2 mm wheal shows a low tendency or predisposition to produce specific IgE, in a continuum which is the rule in biological phenomena.

The definition of the level of atopy by means of ASPTs is based on the assumption that the size of the wheal observed after the epicutaneous injection of an allergen depends exclusively on the amount of histamine that is produced in the antigen-antibody (Ag-Ab) reaction [3]. This assumption would be true if, and only if, "histamine skin reactivity" (HSR) i.e. the size of the wheal produced by a prick test performed with a given solution of histamine $($ e.g. $10 \mathrm{mg} / \mathrm{ml})$ were the same in different people, regardless of age, geographical settings, etc. Several epidemiological data have demonstrated that this is not the case. First of all, individual HSR values in the numerous population studies are fairly evenly spread on either side of the mean (plus or minus 20-30 \%) [4-9]. Moreover, in a number of epidemiological studies, HSR varied significantly in different populations [10-12], and was even found to increase over time within the same geographical setting [5,6]. To what extent HSR variability affects the results of the ASPT has not been previously discussed in any depth and is virtually ignored in various "official position papers" on skin prick testing where histamine is often vaguely treated as the "positive control" [1]. This gap in our knowledge needs to be filled and the consequences at the epidemiological and clinical level of HSR variability on what we define as "atopy" deserve a full and exhaustive discussion.

\section{Histamine Wheal}

Histamine is synthesized above all by mast cells, and when released it causes smooth muscle cell contraction, vasodilatation, increased vascular permeability, inter-cell neurotransmission and nociceptive nerve fiber stimulation. These are some of the numerous pathways that contribute to the formation of a local skin wheal in the presence of histamine, regardless of whether this mediator is released as a consequence of a local $\mathrm{Ag}-\mathrm{Ab}$ reaction (specific IgE antibodies bound to cell membrane cross-links with specific allergens) or is directly injected in the skin. Histamine is metabolized by a deamino-oxidase (DAO, former name: histaminase) that is stored in epithelial cells and is the main factor underlying the balance between histamine production and degradation. A delay in histamine degradation due to impaired enzymatic DAO function has been reported: the defect may be caused by either genetic or acquired (in most cases reversible) factors [13]. Various single-nucleotide polymorphisms in the human DAO gene, located on chromosome $7 \mathrm{q} 35$, are associated with clinical conditions, such as food allergies, and represent a potential genetic background of a reduced or accelerated histamine metabolism [14-16]. A peculiar and interesting case is the rare and complex McCuneAlbright syndrome, which is characterized by precocious puberty and café-au-lait spots and is caused by a genetic mutation encoding a 
stimulatory G protein (G5alpha): all the patients with the syndrome display marked skin reactivity to histamine [17].

\section{Histamine Skin Reactivity in Epidemiology}

Although the histamine prick test is highly reproducible in the same patient [18], its inter-individual variability is considerable. In one large epidemiological study [6], skin prick tests with a $10 \mathrm{mg} / \mathrm{ml}$ histamine solution performed in unselected 9-year-old school children produced a wheal with a 4.5-5.5 $\mathrm{mm}$ diameter and a standard deviation of $0.8-1.0 \mathrm{~mm}$ : the spread of values was such that there was a large overlap in the size of wheals produced by a $1 \mathrm{mg} / \mathrm{ml}$ histamine solution ( $30 \%$ of children were "low responders"). Similar results were obtained by Scherer et al. [18] who, using a very precise technique for a histamine intradermal skin test, found a high reproducibility in the same individuals but a considerable inter-individual variability (standard deviations of the order of $50 \%$ of the mean, with some patients developing a wheal three times as large as other patients). Similar results were obtained by Van Gysell et al. [8]

Histamine-induced skin wheals have been reported to be larger i) in male than in female subjects in some studies [7] though not in others $[5,6,8]$, ii) in the upper back than in the arm [19], iii) in younger than in older subjects [20] and iv) in subjects with higher body mass index [21]. Lower HSR is reported to be associated with some environmental factors, such as passive smoking exposure and sun-exposed skin areas $[9,22]$. Many studies contain data that partially explain this biological HSR variability: differences in "histamine releasability" from mast cells, the relative inefficiency of enzymes responsible for histamine inactivation, variations in the density of mast cell or $\mathrm{H} 1$ receptors, etc. [3]; however, to our knowledge, this variability has not yet been systematically investigated in a human model.

HSR does not appear to be a fixed parameter in humans: three studies, in which cohorts of children aged 9 or 13 years were examined in the same geographical setting at an interval of 13 or 16 years, revealed a significant increase in HSR, which rose approximately 5-7\% per year $[5,6]$. As these studies were performed at the end of the last century and have not been repeated since, it is impossible to know whether this increase is a persisting trend.

One issue that is highly relevant to our discussion is the marked inter-population variability in HSR. This finding first emerged from the work conducted by Dreborg et al. [10], who found a significant difference in histamine-induced wheals of small but thoroughly studied populations from Berlin and Uppsala. Differences of HSR between populations have been found by Riihjavik et al. [23] and by von Mutius et al. [24]. Subsequently, in a large international epidemiological study, we measured the skin wheals elicited by histamine pricked at two concentrations in a total of approximately 800 unselected school children from Italy, Poland and Libya, and found that HSR differed significantly in these three countries: the analysis of the slopes of the regression lines for histamine concentrations in relation to the wheal sizes demonstrated that the mean concentration of injected histamine required to elicit a skin wheal of $3 \mathrm{~mm}$ in diameter was $8.8 \mathrm{mg} / \mathrm{ml}$ in Libya, $29.5 \mathrm{mg} / \mathrm{ml}$ in Italy and $102.1 \mathrm{mg} / \mathrm{ml}$ in Poland [11].

Interestingly, we found that these differences in HSR markedly affected the amount of specific IgE required to produce a wheal of at least $3 \mathrm{~mm}$ in an ASPT (the "atopic person" definition threshold). For pricks performed with Dermatophagoides pteronyssinus, the amount of $\operatorname{IgE}(\mathrm{in} \mathrm{ku} / \mathrm{l}$ ) was 1.25 in Libyan children, 11.51 (i.e. ten times higher) in Polish children, and 3.47 in Italian children [11]. When we subsequently subdivided all the children (regardless of nationality) according to their HSR, we found that the amount of serum-specific IgE required to induce a positive prick test was approximately eight times higher (16.4 vs $2.2 \mathrm{ku} / \mathrm{l}$ for Dermatophagoides pteronyssinus) in children with "'low" histamine skin reactivity (less than the 50th percentile of the overall population) than in children with "high" histamine skin reactivity (over the 50th percentile) [12]; the differences between high and low histamine responders would have been considerably greater had we compared subjects with this parameter under the 10th percentile with those over the 90th percentile [12].

Comparable results have recently been reported by Dreborg et al. who found, when they reanalyzed the data of the experiments conducted in 1985 in Berlin and Uppsala, which the concentration of allergen required to produce a certain skin wheal was 15 times higher in the population that had significantly lower HSR (Berlin) [25].

\section{The Clinical Meaning of Histamine Skin Reactivity}

All the aforementioned data indicate that high HSR "produces" larger or an increased number of positive ASPTs, because even a small amount of specific IgE reacting with the appropriate antibody produces an amount of histamine that is sufficient for a $3 \mathrm{~mm}$ wheal. This interpretation may, however, also be inverted: allergen responsiveness as measured by skin prick tests (atopic state) may result in increased histamine skin sensitivity, a hypothesis backed by numerous authors. For example, Stuckey et al. reported that allergen responsiveness is related to high sensitivity to histamine $(\mathrm{p}<0.001)$, an association that is independent of IgE [26]. Van Gysell et al. [8] found that multiplysensitized children had significantly larger histamine-induced wheal sizes than non-sensitized children. Yet other authors interpreted their data in a comparable fashion $[7,9,27]$.

There is also an intermediate position held by some authors who, being fully aware of the existence of the two determinants of skin wheal dimensions in ASPT (i.e. the amount of specific $\operatorname{IgE}$ and "histamine skin sensitivity"), elaborated complex statistical methods designed to remove the contribution of histamine skin reactivity and hence to correct for the "observed wheal sizes" and avoid "atopy misclassification" [28]. Obviously this approach is based on the assumption that HSR is a "complication" (a disturbing component of the wheal) in the clinical assessment of "true atopy" in patients.

In practice, we may agree that the implications of all the aforementioned data and interpretations are identical: a $3 \mathrm{~mm}$ ASPT wheal may be elicited in any individual (who would consequently be classified as atopic) either by producing several ku of specific IgE per litre of serum or by means of a high HSR.

Although these two extremes are combined in the vast majority of patients, we may hypothesize the existence of two types of patients : one type in whom "atopy" is mainly the result of increased levels of specific IgE antibodies, and another type in whom positive ASPRs are mainly the result of a marked reactivity to even small amounts of histamine. These distinctions are important on clinical grounds: when we treat a patient whose clinical symptoms are generated above all by exposure (through contact, ingestion or inhalation) to known antigens, we find ourselves within a medical context whose study goes back decades and whose underlying enemies we are generally aware of, including the mechanisms of action, preventive strategies, effective immunological and pharmaceutical therapies that need to be undertaken. If we exclude cases suffering from severe poly- 
sensitization, we are confident that an effective treatment for such patients is readily available.

The opposite is instead often true of patients in whom "atopy "mainly depends on marked histamine reactivity. Current knowledge of this biological characteristic is very limited and we are just beginning to understand that this parameter is likely to be affected not only by genetic but also environmental factors, as is clearly suggested by the marked differences between countries and changes over time within the same geographical setting.

Despite not yet being proven, it is reasonable to believe that hyperreactivity to histamine measured in the skin is the same as that in other parts of the organism, particularly in all kinds of mucosae, in which this hyper-reactivity is likely to be triggered by even a small increase in histamine production, thereby causing symptoms such as oedema, extravasation and muscle contraction, which tend to be chronic or recurrent.

In the last decade a syndrome of "histamine intolerance", characterized by symptoms such as gastrointestinal disorders, rhinorrhea and congestion of the nose, headache, urticaria, pruritus and asthma, has been described and investigated [13]: its etiology is complex and supposedly dependent on an imbalance between histamine availability (production or ingestion) and its enzymatic metabolic degradation. The possible connections between "histamine intolerance" and increased "histamine skin reactivity" are evident.

"Histamine intolerance" is only one of the many syndromes recently discussed in the literature that are characterized by recurrent or chronic symptoms. The many other such syndromes include multiple chemical sensitivities, chronic fatigue syndrome, fibromyalgia, chronic hypoglycemia, irritable bowel syndrome, gulf war syndrome and sick building syndrome [29]. Each of these clinical entities is the result of efforts made by practitioners and investigators to label different groups of patients that share a specific, though ill-defined, hyper-sensitivity characterized by exposure to "irritating" environmental factors that result in a range of chronic or recurrent groups of symptoms (gastrointestinal, cutaneous, respiratory and neurological, particularly headache) [30]. The etiology of these conditions not only tends to be obscure or poorly defined, but the symptoms associated with each largely overlap. Indeed, the number of labels that have been used to describe them documents the remarkably high number of medical encounters in which the symptoms reported by individuals do not fall within standard medical conditions, or in which the clinical examination does not correspond to any morphological or laboratory findings.

\section{Conclusion}

We do not know whether the systematic measurement of HSR may help to create a rational approach to these varied groups of patients. We do, however, believe that an easily measured, clinically meaningful biological parameter may contribute to our understanding of the wide and slippery field of unexplained chronic or recurrent clinical symptoms.

\section{References}

1. Bousquet J, Heinzerling L, Bachert C, Papadopoulos NG, Bousquet PJ, et al. (2012) Practical guide to skin prick tests in allergy to aeroallergens. Allergy 67: 18-24.

2. Ruëff F, Bergmann KC, Brockow K, Fuchs T, Grübl A, et al. (2011) [Skin tests for diagnostics of allergic immediate-type reactions. Guideline of the
German Society for Allergology and Clinical Immunology]. Pneumologie 65: 484-495.

3. Clough GF, Lucas JS (2007) Reinventing the weal? Clin Exp Allergy 37: 1-3.

4. Malling HJ (1984) Skin prick testing and the use of histamine references. Allergy 39: 596-601.

5. Ronchetti R, Pia Villa M, Ciofetta G, Barreto M, Falasca C, et al. (2001) Changes over 13 years in skin reactivity to histamine in cohorts of children aged 9-13 years. Allergy 56: 436-441.

6. Ronchetti R, Villa MP, Pagani J, Martella S, Guglielmi F, et al. (2003) Immediate skin reactivity to histamine and to allergens in cohorts of 9year-old schoolchildren studied 16 years apart. Clin Exp Allergy 33: 1232-1237.

7. Bordignon V, Burastero SE (2006) Age, gender and reactivity to allergens independently influence skin reactivity to histamine. J Investig Allergol Clin Immunol 16: 129-135.

8. Van Gysel D, Govaere E, Verhamme K, Doli E, De Baets F (2007) The influence of atopic status and potential risk factors for sensitization on histamine skin reactivity in unselected Belgian children. Pediatr Dermatol 24: 363-368.

9. Kidon MI, See Y, Bun CY, Goh A, Chay OM, et al. (2004) Bimodal skin reactivity to histamine in atopic children in Singapore: influence of specific sensitizations. Pediatr Allergy Immunol 15: 545-550.

10. Dreborg S, Basomba A, Belin L, Durham S, Einarsson R, et al. (1987) Biological equilibration of allergen preparations: methodological aspects and reproducibility. Clin Allergy 17: 537-550.

11. Ronchetti R, Villa MP, Bohmerova Z, Martella S, Falasca C, et al. (2004) Skin reactivity to histamine and codeine in unselected 9-year-old children from Italy, Poland and Libya. Int Arch Allergy Immunol 135: 136-142.

12. Ronchetti R, Villa MP, Rennerova Z, Haluszka J, Dawi EB, et al. (2005) Allergen skin weal/radioallergosorbent test relationship in childhood populations that differ in histamine skin reactivity: a multi-national survey. Clin Exp Allergy 35: 70-74.

13. Maintz L, Novak N (2007) Histamine and histamine intolerance. Am J Clin Nutr 85: 1185-1196.

14. Petersen J, Raithel M, Schwelberger HG (2005) Characterisation of functional polymorphisms of the human diamine oxidase gene. Inflamm Res 54 Suppl 1: S58-59.

15. Raithel M, Küfner M, Ulrich P, Hahn EG (1999) The involvement of the histamine degradation pathway by diamine-oxidase in manifest gastrointestinal allergies. Inflamm Res 48: S75-S76.

16. Schwelberger HG (2004) Diamine oxidase (DAO) enzyme and gene. In: Falus A, editor: Histamine: Biology and Medical Aspects. Spring Med Publishing, Budapest.

17. Jacobson JD, Turpin AL, Sands SA (2013) Allergic manifestations and cutaneous histamine responses in patients with McCune Albright syndrome. World Allergy Organ J 6: 9.

18. Scherer K, Grize L, Schindler C, Surber C, Bircher AJ (2007) Reaction pattern to histamine and codeine in a human intradermal skin test model. Clin Exp Allergy 37: 39-46.

19. Yuenyongviwat A, Koonrangsesomboon D, Sangsupawanich P (2012) Comparison of skin test reactivity to histamine on back and forearm in young children. Asian Pac J Allergy Immunol 30: 301-305.

20. Niemeijer NR, de Monchy JG (1992) Age-dependency of sensitization to aero-allergens in asthmatics. Allergy 47: 431-435.

21. Kang JW, Lee KH, Hong SC, Lee HS, Lee J, et al. (2015) Histamine skin reactivity increases with body mass index in Korean children. Int J Pediatr Otorhinolaryngol 79: 111-114.

22. King MJ, Phillips SE, Lockey RF (2014) Effect of photoaging on skin test response to histamine independent of chronologic age. Ann Allergy Asthma Immunol 113: 647-651.

23. Riikjärv MA, Julge K, Vasar M, Bråbäck L, Knutsson A, et al. (1995) The prevalence of atopic sensitization and respiratory symptoms among Estonian schoolchildren. Clin Exp Allergy 25: 1198-1204. 
Citation: Ronchetti R, Barreto M (2015) The Clinical Meaning of Histamine Skin Reactivity. J Allergy Ther 6: 227. doi:

24. von Mutius E, Weiland SK, Fritzsch C, Duhme H, Keil U (1998) Increasing prevalence of hay fever and atopy among children in Leipzig, East Germany. Lancet 351: 862-866.

25. Dreborg S (2015) Allergen skin prick test should be adjusted by the histamine reactivity. Int Arch Allergy Immunol 166: 77-80.

26. Stuckey MS, Witt CS, Schmitt LH, Warlow R, Lattimore M, et al. (1985) Histamine sensitivity influences reactivity to allergens. J Allergy Clin Immunol 75: 373-376.

27. Halász MR, Gonsales SL, Solé D, Naspitz CK (1997) Specific sensitization to Dermatophagoides pteronyssinus and cutaneous reactivity to histamine in Brazilian children. J Investig Allergol Clin Immunol 7: 98-102.
28. Zhang H, Karmaus W, Gan J, Bao W, Zhao YD, et al. (2011) Adjusting wheal size measures to correct atopy misclassification. Int J Gen Med 4: 597-606.

29. Genuis SJ (2010) Sensitivity-related illness: the escalating pandemic of allergy, food intolerance and chemical sensitivity. Sci Total Environ 408: 6047-6061.

30. Kipen HM, Fiedler N (2002) Environmental factors in medically unexplained symptoms and related syndromes: the evidence and the challenge. Environ Health Perspect 110: 597-599. 